\title{
The potentiostatic transient for 3D nucleation with diffusion-controlled growth: theory and experiment for progressive nucleation
}

\author{
Edward Matthijs ${ }^{a}$, Steven Langerock ${ }^{\text {a, Emilia Michailova }}{ }^{\text {b }, ~ L u c ~ H e e r m a n ~}{ }^{\text {a,* }}$ \\ a Department of Chemistry, Afdeling Coordinatiechemie, Katholieke Universiteit Leuven, Celestijnenlaan 200G, B3001 Heverlee, Leuven, Belgium \\ ${ }^{\mathrm{b}}$ Institute of Physical Chemistry, Bulgarian Academy of Sciences, Acad. G. Bonchev Street bl. 11, 1113 Sofia, Bulgaria
}

Received 1 December 2003; received in revised form 11 March 2004; accepted 12 March 2004

Available online 24 June 2004

\begin{abstract}
The theory of the potentiostatic transient for 3D nucleation with diffusion-controlled growth is discussed. It is shown that the theoretical model of Mirkin and Nilov [J. Electroanal. Chem., 283 (1990) 35] and Heerman and Tarallo [J. Electroanal. Chem., 470 (1999) 70] predicts too high values of the current, which becomes very apparent for high values of the site density and low values of the nucleation rate constant (progressive nucleation). For example, the model then predicts that the current in the limit of long times will be higher than the Cottrell limit by a factor of $4 / 3$ which is physically unacceptable. Therefore, a modification to this model is proposed which is based on a careful analysis of the Kolmogorov-Avrami theorem. The "extended area" in the KolmogorovAvrami theorem includes contributions from "phantom nuclei" that are born inside already existing zones but do not exist physically. This is necessary to preserve the randomness of the system and allows the correct calculation of the appearance rate of the nuclei and the nucleus saturation density. The "extended current", defined in analogy with the "extended area", then also attributes current to the phantom nuclei. It follows that the ratio $j_{\mathrm{ex}}(t) / \theta_{\mathrm{ex}}(t)$ which appears in the model of Mirkin and Nilov and Heerman and Tarallo does not correspond to the actual number of nuclei formed on the electrode. Therefore, the "extended quantities" in this ratio must be replaced with quantities that relate directly to the real number of clusters (this implies what is fairly obvious, that the appearance rate of the clusters must be calculated first). This makes it is possible to derive an equation that predicts correctly the current in the limits of both short and long times which is directly linked to the $N_{\mathrm{a}}(t)$ vs. time relation (where $N_{\mathrm{a}}(t)$ is the actual number of nuclei on the electrode). Experiments for the nucleation of silver on glassy carbon electrodes, with the simultaneous recording of both $j(t)$ vs. time and $N_{\mathrm{a}}(t)$ vs. time relations, are described. The experimental results obtained from the transients and the direct visual counting of nuclei are compared with the theoretical predictions.
\end{abstract}

(C) 2004 Elsevier B.V. All rights reserved.

Keywords: Nucleation; Transient; Diffusion-controlled growth

\section{Introduction}

Several models have been proposed for the theoretical description of the potentiostatic transient for 3D nucleation on active sites with diffusion-controlled growth [1-12]. All these models describe the rate law for the formation of nuclei on a finite number of active sites, which are distributed randomly over the electrode surface, by the equation

\footnotetext{
${ }^{*}$ Corresponding author. Tel.: +32-16-327-337; fax: +32-16-327-992.

E-mail address: luc.heerman@chem.kuleuven.ac.be (L. Heerman).
}

$\frac{\mathrm{d} N(t)}{\mathrm{d} t}=\left[N_{0}-N(t)\right] A$,

where $N(t)$ is the nucleus number density, $N_{0}$ the site density and $A$ the nucleation rate constant. Integration of Eq. (1), with the initial condition $N(t)=0$ for $t=0$, then yields the $N(t)$ vs. time relation

$N(t)=N_{0}[1-\exp (-A t)]$,

which has two well-known limiting cases. For "instantaneous nucleation", $N(t)=N_{0} H(t)$ (where $H(t)$ is the Heavyside step function) so that all the nuclei are formed immediately after the application of the potential step. 
For high values of the ratio $N_{0} / A, N_{0}-N(t) \simeq N_{0}$ so that $N(t)=A N_{0} t=I_{\text {st }} t$, where $I_{\mathrm{st}}=A N_{0}$ is the stationary nucleation rate constant. This limiting case corresponds to a constant nucleation rate and the nucleus density increases linearly with time. However, this linear law vastly overestimates the nucleus number density because it neglects the deactivation of active sites by the diffusion fields associated with already existing, growing nuclei ("ingestion" of active sites). This effect leads to a decreasing appearance rate of the nuclei and hence to the formation of a finite number of clusters, the nucleus saturation density. This paper discusses theoretical models for the potentiostatic transient with special emphasis on the relation between the current and the experimental nuclei vs. time curve, i.e. the real appearance rate of the nuclei.

\section{The concept of planar diffusion zones}

Theoretical models for the potentiostatic transient are based on the concept of planar diffusion zones. In the case of multiple nucleation and growth, the flux to each nucleus is shielded by the surrounding nuclei that form a "Faraday-diffusional cage" [13]. The flux to the nuclei, and hence their growth rate, decreases and the mass transfer regime changes from hemispherical to planar diffusion. The concept of "planar diffusion zones" was introduced to obtain approximate solutions for the resulting diffusion problem that cannot be solved analytically. A planar diffusion zone $S_{\mathrm{d}}(t, u)$ is defined as the equivalent area needed to sustain the growth of the hemispherical nucleus by planar diffusion. The current to a single hemispherical nucleus can be written in a form that presents an analogy with a planar Cottrell current to this fictive circular area on the electrode. For sufficiently large overvoltages, $|\eta| \rightarrow \infty$, the current to a single nucleus born at time $u$ is given by

$I(t, u)=z F D \bar{c} \sqrt{\pi D} k \sqrt{t-u}=z F D \bar{c} \frac{\pi D k(t-u)}{\sqrt{\pi D(t-u)}}$,

where $k=\sqrt{8 \pi V_{\mathrm{m}} \bar{c}}\left(V_{\mathrm{m}}\right.$ is the molar volume of the deposited phase). The area of the planar diffusion zone is given by $S_{\mathrm{d}}(t, u)=\pi D k(t-u)$ and the height of the corresponding diffusion cylinder by $\sqrt{\pi D(t-u)}$. The problem of interference of the diffusion fields of the nuclei then can be described by the overlap of the associated diffusion zones, which forms the basis of the "standard model" of Scharifker and Mostany [3]. The concept of planar diffusion zones has been amply discussed in the literature and more details can be found in the original papers $[2,3,8,12]$.

\section{Nucleation exclusion zones}

For diffusion control, a zone depleted of electroactive material develops around the growing nuclei. Therefore the appearance rate of the nuclei, which is a function of the local concentration of metal ions [14-17], decreases in the vicinity of already existing clusters. Free sites close to a growing nucleus can be deactivated and this effect is referred to as "ingestion of active sites" [2,4,14,16-21]. Thus, it is necessary to modify Eq. (1) and, quite generally, the real appearance rate of the nuclei $\mathrm{d} N_{\mathrm{a}} / \mathrm{d} t^{1}$ can be written as

$\frac{\mathrm{d} N_{\mathrm{a}}(t)}{\mathrm{d} t}=\left[N_{0}-N_{\mathrm{a}}(t)-N_{\text {ing }}(t)\right] A=\left[1-\frac{N_{\mathrm{a}}(t)+N_{\text {ing }}(t)}{N_{0}}\right] A N_{0}$,

where $N_{\text {ing }}(t) \geqslant 0$ is the number density of ingested sites. Ingestion of active sites evidently results in a decrease of the appearance rate of the nuclei and after a sufficiently long time, all the sites will be occupied by growing nuclei or deactivated by ingestion so that the appearance rate of the nuclei tends to zero. This results in the formation of a finite number of nuclei, the "nucleus saturation density". According to Eq. (1), as $t \rightarrow \infty$, the nucleus density will always become equal to $N_{0}$, i.e. the site density. However, it is readily anticipated that the nucleus saturation density will be determined mainly by the effect of ingestion if $I_{\mathrm{st}}=A N_{0}$ is very low compared with the growth rate of the nuclei. The nucleus saturation density is an experimentally observable quantity [22-26] so that predictions of theory can be compared directly with the results of visual observation (including techniques such as AFM, STM, ... [27,28]). Experimental evidence for the effect of ingestion has been obtained also from studies of the spatial distribution of the nuclei over the electrode surface [2,14-17,21,23-26]. Ingestion of active sites leads to a decrease of the probability of nucleation in the vicinity of growing nuclei and therefore disrupts the random distribution of nuclei. Thus, histograms of experimentally determined nearest neighbour distributions of nuclei offer a direct proof of the inhibition of nucleus formation in the vicinity of an existing, growing nucleus. The effect of ingestion has been studied also by several authors by means of Monte-Carlo simulations of the nucleation kinetics [2,15-17,29,30].

Exact expressions have been presented in the literature for the probability of nucleus formation as a continuous function of distance from an already existing single nucleus growing under diffusion control, including the effects of mixed kinetics and ohmic drop [14-17]. These equations provide a better understanding of the physical reason for the effect of ingestion but evidently they do not take into account the interference of

\footnotetext{
${ }^{1}$ In this paper $\mathrm{d} N_{\mathrm{a}} / \mathrm{d} t$ (Eq. (4)) is called the appearance rate of the nuclei to avoid confusion with $\mathrm{d} N / \mathrm{d} t$ (Eq. (1)). However, an experimentalist who would observe the formation of clusters by direct visual observation no doubt would refer to $\mathrm{d} N_{\mathrm{a}} / \mathrm{d} t$ as the "nucleation rate".
} 
diffusion fields. Therefore, several authors introduced the concept of "exclusion zones" as a useful simplification [2,16-21]. An exclusion zone is defined as a planar circular area $S_{\mathrm{ex}}(t, u)$ that develops around a growing nucleus, from the moment of its birth, comparable with a planar diffusion zone. The probability of nucleus formation is zero inside the exclusion zone (fully arrested nucleation) and retains its initial value outside the zone. For diffusion-controlled growth the radius of the exclusion zones increases with the square root of time from the moment of their birth. It has been proposed to put $S_{\mathrm{ex}}(t, u)=S_{\mathrm{d}}(t, u)$, i.e. to identify the area of the exclusion zones with the area of the planar diffusion zones. This may look at first sight as a drastic oversimplification but this assumption is reasonable because a planar diffusion zone can be considered as an area where the surface concentration of electroactive material, and hence the probability of nucleus formation, is zero. The situation is even more complex because, as Milchev [14, p. 248] has pointed out, in principle one must take into account also the effect of the reduced concentration and overpotential in the vicinity of a single growing cluster on the size of the critical nuclei. Therefore, it must be borne in mind that there is no real theoretical justification to write $S_{\mathrm{ex}}(t, u)=S_{\mathrm{d}}(t, u)$ and ultimately the validity of this assumption can be checked only by comparing experimental $N_{\mathrm{a}}(t)$ vs. time curves with the predictions of theory.

\section{Appearance rate of nuclei for progressive nucleation}

As the nuclei grow, the diffusion-exclusion zones will overlap and cover a fraction $\theta(t)$ of the electrode surface. At this point it is advantageous to introduce the factor $\alpha=\pi D k N_{0} / A$, where $\pi D k$ is the growth rate constant of the zones. Obviously, $\alpha \rightarrow 0$ for the limiting case of instantaneous nucleation $\left(N_{0} / A \rightarrow 0\right)$ and $\alpha \rightarrow \infty$ for the limiting case of progressive nucleation $\left(N_{0} / A \rightarrow \infty\right)$. For progressive nucleation $\left(N_{0} \rightarrow \infty\right.$, which de facto corresponds to a homogeneous surface) it is possible to write the simple relation

$N_{0} \theta(t)=N_{\mathrm{a}}(t)+N_{\text {ing }}(t)($ for all times $t>0)$,

where $N_{0} \theta(t)$ is the total number of sites within the zones and the right hand side follows from the fact that all these sites are deactivated either by direct occupation of nuclei or by ingestion. Then it follows immediately that in this case

$\frac{\mathrm{d} N_{\mathrm{a}}(t)}{\mathrm{d} t}=[1-\theta(t)] A N_{0}$

and the appearance rate of the real nuclei is given by the product of the free area and the nucleation rate $A N_{0}$ (which of course follows from Eq. (1) with $N_{0}-N(t) \simeq N_{0}$ ). Clearly, $A N_{0}$ describes the formation rate of both the real nuclei and the "phantom nuclei", defined as nuclei that are born inside the exclusion zones but do not exist physically (evidently, for $t \rightarrow 0$, $\theta(t) \rightarrow 0$ and $\left.\mathrm{d} N_{\mathrm{a}}(t) / \mathrm{d} t \rightarrow A N_{0}\right)$. The covered fraction $\theta(t)$ can be calculated by the Kolmogorov-Avrami equation [31-33]

$\theta(t)=1-\exp \left[-\theta_{\text {ex }}(t)\right]$,

where $\theta_{\mathrm{ex}}(t)$ is the extended area, i.e. the total area that would be covered if the zones do not overlap (for $t \rightarrow \infty, \theta_{\mathrm{ex}}(t) \rightarrow \infty$ so that the extended area is not really a fractional quantity). The Kolmogorov-Avrami formula is a result of geometrical statistics that describes, for the case considered here, the overlap of randomly distributed, growing circular disks on a plane surface. To preserve the randomness of the system, the contribution of the phantom nuclei must be taken into account in the calculation of the extended area. This point has often been a source of confusion and was recently discussed in depth by Tomellini and coworkers [34-36]. Thus, the extended area must be calculated as

$$
\begin{aligned}
\theta_{\mathrm{ex}}(t) & =\int_{0}^{t} S_{\mathrm{d}}(t, u) \frac{\mathrm{d} N \mathrm{~d}(u)}{\mathrm{d} u} \mathrm{~d} u \\
& =\pi D k A N_{0} \int_{0}^{t}(t-u) \mathrm{d} u=\frac{\pi D k A N_{0}}{2} t^{2} .
\end{aligned}
$$

Then, the Kolmogorov-Avrami equation rigorously describes the expansion of $\theta(t)$ with time on the strict condition, however, that the zones associated with phantom nuclei do not contribute to the covered area. This implies that the zone associated with a phantom nucleus, which is born at a later time inside an already existing zone, can never outgrow its "parent". This condition is not fulfilled for the case considered here (growth controlled by diffusion) which corresponds to a decelerating growth rate of the zones $\left(S_{\mathrm{d}}(t, u) \approx(t-u) ; \quad r_{\mathrm{d}}(t, u) \approx \sqrt{t-u}\right)$. However, computer simulations [30,37-39] have shown that the error introduced is small (less than $2 \%$ ) and further it is assumed that the Kolmogorov-Avrami equation can also be used to describe the overlap of zones for growth controlled by diffusion. Furthermore, as already noted, ingestion of sites disrupts the random distribution of nuclei and introduces some ordering in the spatial distribution of the nuclei but this does not affect the validity of the Kolmogorov-Avrami equation because the sites on the free area remain randomly distributed.

Combination of Eqs. (6)-(8) yields the expression for the real appearance rate of the nuclei as

$$
\frac{\mathrm{d} N_{\mathrm{a}}(t)}{\mathrm{d} t}=A N_{0} \exp \left(-\frac{\pi D k A N_{0}}{2} t^{2}\right)=A N_{0} \exp \left(-a^{2} t^{2}\right)
$$

with $a=\sqrt{\pi D k A N_{0} / 2}$. Integration of Eq. (9) yields 


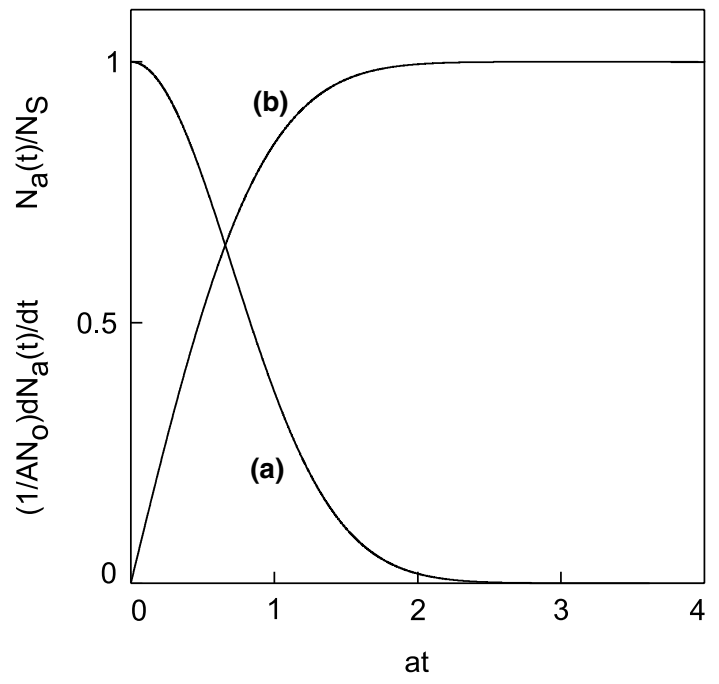

Fig. 1. Plots of (a) $\left(1 / A N_{0}\right) \mathrm{d} N_{\mathrm{a}}(t) / \mathrm{d} t$ vs. at (Eq. (9)) and (b) $N_{\mathrm{a}}(t) / N_{\mathrm{S}}$ vs. at (Eq. (10)) with $N_{\mathrm{S}}=\sqrt{A N_{0} / 2 k D}$ (Eq. (11)).

$$
\begin{aligned}
N_{\mathrm{a}}(t) & =A N_{0} \int_{0}^{t} \exp \left(-a^{2} u^{2}\right) \mathrm{d} u \\
& =\frac{A N_{0}}{a} \int_{0}^{a t} \exp \left(-z^{2}\right) \mathrm{d} z=N_{\mathrm{S}} \operatorname{erf}(a t),
\end{aligned}
$$

where the nucleus saturation number density is given by

$N_{\mathrm{S}}=\frac{\pi^{1 / 2}}{2} \frac{A N_{0}}{a}=\sqrt{\frac{A N_{0}}{2 k D}}$.

A graph of $\mathrm{d} N_{\mathrm{a}}(t) / \mathrm{d} t$ and of the $N_{\mathrm{a}}(t) / N_{\mathrm{S}}$ vs. time relation is shown in Fig. 1.

This shows that the nucleus saturation density depends on two opposing quantities. An increase of $A N_{0}$ (larger overpotential), for fixed experimental conditions (constant $k D$ ), will result in a larger value of the nucleus saturation density (more but smaller nuclei). For a constant value of $A N_{0}$ (constant overpotential), an increase of $k D$ (increasing the concentration) will result in faster growth of the nuclei but smaller values of $N_{\mathrm{S}}$.

\section{Growth of stable clusters and overlap of diffusion zones}

The growth of stable clusters and the interference of their diffusion fields will gradually result in the development of a planar diffusion current. Therefore, the current density for multiple nucleation and growth can be written, quite generally, in the form $[8,9]$

$j(t)=z F D \bar{c} \frac{\theta(t)}{\bar{\partial}(t)}=\frac{z F D \bar{c}}{\bar{\partial}(t)}\left\{1-\exp \left[-\theta_{\mathrm{ex}}(t)\right]\right\}$,

upon which all models using the concept of planar diffusion zones are based. In Eq. (12) $\theta(t)$ is the fraction of the electrode area covered by planar diffusion zones and $\bar{\partial}(t)$ is the thickness of the diffusion layer that is sup- posed to be uniform. For the limit of very long times the diffusion layer thickness has a clear physical meaning and must tend to the Cottrell value so that, for $t \rightarrow \infty$, $\left.\bar{\partial}(t)\right|_{t \rightarrow \infty} \rightarrow \sqrt{\pi D t}$. For very short times, $t \rightarrow 0$, both overlap of diffusion zones and ingestion of sites can be neglected and the current density, with $\mathrm{d} N / \mathrm{d} t=\mathrm{d} N_{\mathrm{a}} / \mathrm{d} t$, can be calculated from

$\left.j(t)\right|_{t \rightarrow 0}=\int_{0}^{t} I(t, u) \frac{\mathrm{d} N(u)}{\mathrm{d} u} \mathrm{~d} u$,

so that for progressive nucleation with a stationary nucleation rate $A N_{0}$

$$
\begin{aligned}
\left.j(t)\right|_{t \rightarrow 0} & =z F D \bar{c} \sqrt{\pi D} k A N_{0} \int_{0}^{t} \sqrt{t-u} \mathrm{~d} u \\
& =\frac{2}{3} z F D \bar{c} \sqrt{\pi D} k A N_{0} t^{3 / 2} .
\end{aligned}
$$

As $t \rightarrow 0,\left.\theta(t)\right|_{t \rightarrow 0} \rightarrow \theta_{\text {ex }}(t)=a^{2} t^{2}=\pi D k N_{0} / 2$ and inserting this and Eq. (14) into Eq. (12) shows that $\bar{\partial}(t)_{t \rightarrow 0} \rightarrow(3 / 4) \sqrt{\pi D t}$. Thus, the problem is to find a function of time that is equal to $3 / 4$ for very short times and that becomes equal to 1 for the limit of very long times.

In its general formulation, the "standard model" of Scharifker-Mostany [3] puts $\bar{\partial}(t)=\sqrt{\pi D t}$ at all times $t$ so that the current density is simply given by the Cottrell current to the covered fraction of the electrode area. Then, for the case of progressive nucleation, the current density is given by

$j(t)=\frac{z F D \bar{c}}{\sqrt{\pi D t}} \theta(t)=\frac{z F D \bar{c}}{\sqrt{\pi D t}}\left[1-\exp \left(-a^{2} t^{2}\right)\right]$.

It is important to note that Eq. (15) implicitly takes into account the formation of a finite number of nuclei because phantom nuclei do not contribute to the covered area. However, this equation evidently cannot describe correctly the initial, rising part of the current transient.

Heerman and Tarallo [8] argued that the diffusion layer thickness should not only be a function of the time variable $t$ but also of the nucleation frequency, i.e., the appearance rate of the nuclei. They proposed to calculate the diffusion layer thickness from the equation

$j_{\mathrm{ex}}(t)=z F D \bar{c} \frac{\theta_{\mathrm{ex}}(t)}{\bar{\partial}(t)}$,

where the extended current density is defined as $[4,8,12]$

$j_{\mathrm{ex}}(t)=\int_{0}^{t} I(t, u) \frac{\mathrm{d} N(u)}{\mathrm{d} u} \mathrm{~d} u$.

Combination of Eqs. (12) and (16) then yields a simple general equation for the potentiostatic transient

$j(t)=j_{\mathrm{ex}}(t) \frac{\theta(t)}{\theta_{\mathrm{ex}}}=j_{\mathrm{ex}}(t) \frac{1-\exp \left[-\theta_{\mathrm{ex}}(t)\right]}{\theta_{\mathrm{ex}}(t)}$,

which was derived earlier by Mirkin and Nilov [6] using different arguments. However, Eq. (18) breaks down 
completely for large values of $\alpha=\pi D k N_{0} / A$, i.e. for high values of the site density and low values of the nucleation rate constant. For the limiting case of progressive nucleation with a stationary nucleation rate $A N_{0}$ the extended current density, as defined by Eq. (17), is given by

$$
\begin{aligned}
j_{\text {ex }}(t) & =z F D \bar{c} \sqrt{\pi D} k A N_{0} \int_{0}^{t} \sqrt{t-u} \mathrm{~d} u \\
& =\frac{2}{3} z F D \bar{c} \sqrt{\pi D} k A N_{0} t^{3 / 2} .
\end{aligned}
$$

The expression on the right hand side of Eq. (18) is identical with that in Eq. (14), as it should be of course in the limit of very short times, but now remains valid over the whole time domain. This leads to $\bar{\partial}(t) \rightarrow(3 / 4) \sqrt{\pi D t}$ and to the following equation for the current density:

$j(t)=\frac{4}{3} \frac{z F D \bar{c}}{\sqrt{\pi D t}} \theta(t)=\frac{4}{3} \frac{z F D \bar{c}}{\sqrt{\pi D t}}\left[1-\exp \left(-a^{2} t^{2}\right)\right]$.

Eq. (20) correctly describes the current at very short times but fails to predict the expected Cottrell behaviour at times $t>t_{\max }$, i.e. after the current maximum.

The reason why Eq. (18) fails is not difficult to understand. As stated already, the thickness of the diffusion layer should depend on the time variable $t$ and on the nucleation frequency, i.e. on the appearance rate of the real nuclei. The problem is depicted schematically in Fig. 2.

The solid circles in Fig. 2(a) represent the planar diffusion zones associated with real growing clusters and the broken circles, the zones associated with the phantom nuclei which are born inside an already existing "real" zone. The extended area $\theta_{\mathrm{ex}}(t)$ as defined by Eq. (8) is the sum of all these zones when there is no overlap. As discussed in a previous section, the correct applica-
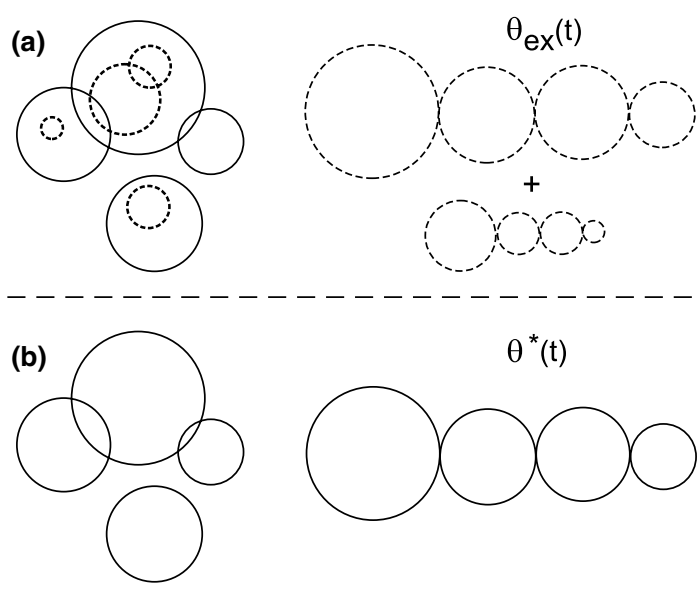

$\theta^{*}(\mathrm{t})$

Fig. 2. Schematic view of the meaning of $\theta_{\mathrm{ex}}(t)$ and $\theta^{*}(t)$. The full circles represent the planar zones associated with the real growing clusters and the broken clusters are the zones associated with phantom nuclei. tion of the Kolmogorov-Avrami formula for the covered area $\theta(t)$ requires that the "phantom zones" are included in the calculation of $\theta_{\mathrm{ex}}(t)$ to preserve the condition of randomness. Then, if the effect of ingestion is taken into account, the calculation of $j_{\mathrm{ex}}(t)$ refers to a hypothetical situation of independent growth but associates a current not only with the real but also with the phantom nuclei.

It is however clear that the definition of $\theta_{\mathrm{ex}}(t)$ is needed only to obtain an analytical expression for the covered area by means of the Kolmogorov-Avrami formula. Therefore it is instructive to consider the case of digital simulation of nucleation and growth where an analytical expression for $\theta(t)$ is not needed. During each slice of time, the area of the already existing zones is updated and a new pair of $x, y$-coordinates is randomly generated on the simulation area. A new nucleus is born only if the corresponding point is located outside the covered area, in agreement with the concept of exclusion zones. If the point is located inside an already existing zone, nothing happens because there is no need to consider phantom zones and the program will simply move to the next trial, i.e. the next slice of time. The corresponding distribution of zones is depicted in Fig. 2(b). Evidently it is not important whether the phantom zones are drawn or not, because they do not contribute to the covered area (as discussed previously, there is a small correction needed for the case of diffusion-controlled growth but this is neglected in this discussion). This also means that the $N_{\mathrm{a}}(t)$ vs. time curve is the same for the situations depicted in Fig. 2(a) and (b).

Thus it is possible to define an area $\theta^{*}(t)$ as

$$
\begin{aligned}
\theta^{*}(t) & =\int_{0}^{t} S_{\mathrm{d}}(t, u) \frac{\mathrm{d} N_{\mathrm{a}}(u)}{\mathrm{d} u} \mathrm{~d} u \\
& =\pi D k A N_{0} \int_{0}^{t}(t-u) \exp \left(-a^{2} u^{2}\right) \mathrm{d} u,
\end{aligned}
$$

which corresponds to the non-overlapped area of the planar diffusion zones associated with the real nuclei. The integral in Eq. (21) is readily evaluated as

$$
\begin{aligned}
\theta^{*}(t) & =2 a^{2} \int_{0}^{t}(t-u) \exp \left(-a^{2} u^{2}\right) \mathrm{d} u \\
& =\sqrt{\pi} a t \operatorname{erf}(a t)-\left[1-\exp \left(-a^{2} t^{2}\right)\right] .
\end{aligned}
$$

A plot of $\theta^{*}(t)$ vs. at is shown in Fig. 3. For $t \rightarrow 0$, $\theta^{*}(t) \rightarrow a^{2} t^{2}$ and for $t \rightarrow \infty, \theta^{*}(t) \rightarrow \sqrt{\pi}$ at what is the expected result for the instantaneous formation of $N_{\mathrm{S}}$ nuclei (for decelerating growth the zones associated with nuclei that appear at later times overhaul the zones associated with nuclei born in the beginning). Then it is also possible to calculate the current density $j^{*}(t)$ as

$j^{*}(t)=\int_{0}^{t} I(t, u) \frac{\mathrm{d} N_{\mathrm{a}}(u)}{\mathrm{d} u} \mathrm{~d} u$. 


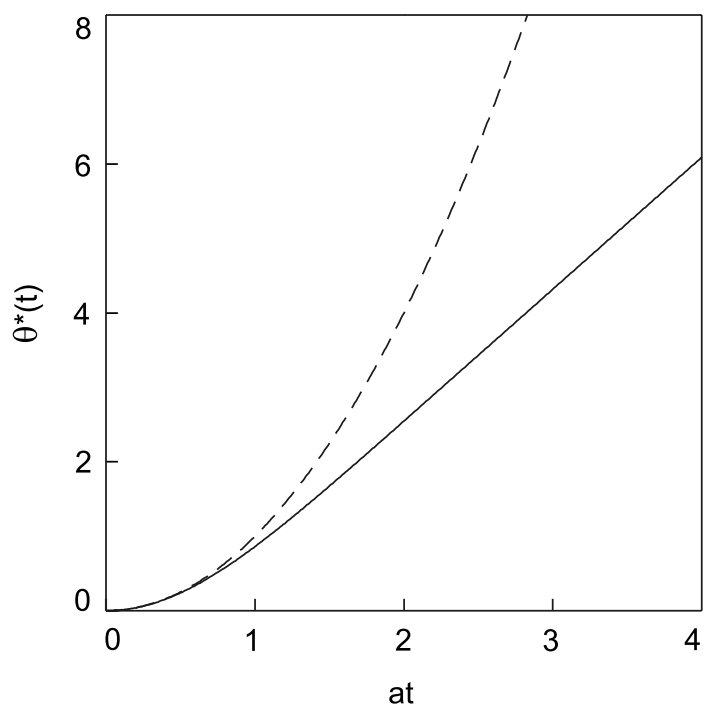

Fig. 3. Plots of $\theta^{*}(t)$ vs. at (Eq. (22)) (solid line) and of $\theta_{\mathrm{ex}}(t)=a^{2} t^{2}$ vs. at (Eq. (8)).

This is the current density associated with the fictive independent growth of the real nuclei if they were to appear according to Eq. (9). For the limiting case of progressive nucleation $j^{*}(t)$ is given by

$j^{*}(t)=z F D \bar{c} \sqrt{\pi D} k A N_{0} \int_{0}^{t} \sqrt{t-u} \exp \left(-a^{2} u^{2}\right) \mathrm{d} u$.

The integral in Eq. (24) can be evaluated only numerically and a plot of $j^{*}(t)$ vs. at is shown in Fig. 4. For $t \rightarrow 0, j^{*}(t) \rightarrow j_{\text {ex }}(t)$ but at later times $j^{*}(t)<j_{\text {ex }}(t)$ because of the decreasing appearance rate of the nuclei; for $t \rightarrow \infty, j^{*}(t) \rightarrow z F D \bar{c} \sqrt{\pi D} k N_{\mathrm{S}} \sqrt{t}$, again corresponding to the instantaneous formation of $N_{\mathrm{S}}$ nuclei.

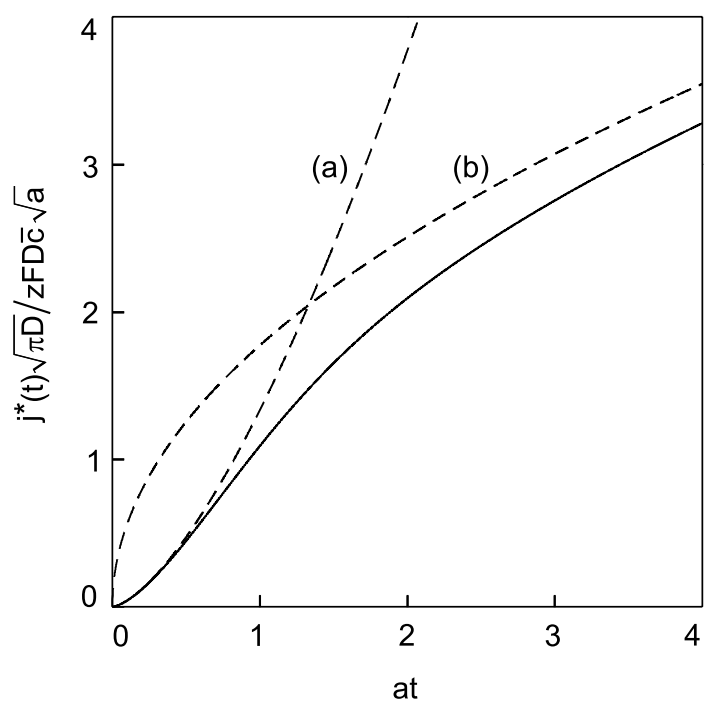

Fig. 4. Plot of $j^{*}(t) \sqrt{\pi D} / z F D \bar{c} \sqrt{a}$ vs. at (solid line). The broken line (a) is a plot of $j_{\mathrm{ex}}(t) \sqrt{\pi D} / z F D \bar{c} \sqrt{a}=4 / 3(a t)^{3 / 2}$, i.e. the extended current density. The broken line (b) is a plot of $j^{*}(t) \sqrt{\pi D} / z F D \bar{c} \sqrt{a}=\sqrt{\pi a t}$, which corresponds to the instantaneous nucleation of $N_{\mathrm{S}}$ nuclei.
It is proposed to calculate the inverse diffusion layer thickness from the ratio $j^{*}(t) / \theta^{*}(t)$. Then the equation for the current density becomes

$j(t)=\frac{j^{*}(t)}{\theta^{*}(t)} \theta(t)=\frac{j^{*}(t)}{\theta^{*}(t)}\left\{1-\exp \left[-\theta_{\mathrm{ex}}(t)\right]\right\}$,

which is the major result of this work. It is readily verified that this equation now correctly predicts the expected behaviour in the limits of both very short and very long times. For $t \rightarrow \infty, \theta(t) \rightarrow 1, j^{*}(t) \rightarrow z F D \bar{c} \sqrt{\pi D} k N_{\mathrm{S}} \sqrt{t}$ and $\theta^{*}(t) \rightarrow \pi D k N_{\mathrm{S}} t$ so that the current tends to the Cottrell value, which is the expected result. For very short times, $j^{*}(t) \rightarrow j_{\mathrm{ex}}(t), \theta^{*}(t) \rightarrow \theta(t)=\theta_{\mathrm{ex}}(t)$ and the current describes correctly the initial part of the transient.

It is worth mentioning that Cao et al. [40] recently reported the results of direct numerical simulation of nucleation and 3D diffusion-controlled growth. The simulations were based on a 3D Green function which allows an exact solution of the mass transfer problem to a very large number of nuclei. This approach calculates the concentration on non-nucleated sites and thus allows in principle the introduction of concentration-dependent nucleation rates. They reported good agreement between theory and simulations for the case of instantaneous nucleation, which shows that the concept of planar diffusion zones is physically acceptable. For finite nucleation rates the results of the simulations were in good agreement with the model of Mirkin and Nilov [6] and Heerman and Tarallo [8]. However, the simulation results as reported in the paper of Cao et al. [40] refer to a $N(t)$ vs. time relation consistent with Eq. (2) and hence neglect the effect of ingestion of active sites. Therefore the good agreement of the simulation results with the predictions of Eq. (20) shows that the physical basis of the overlap model is quite acceptable but this does not imply that these results also correctly describe experimental transients.

\section{Properties of the transient for progressive nucleation}

In dimensionless form the transient depends only on the value of $a=\sqrt{\pi k D A N_{0} / 2}$. A plot of $j(t) \sqrt{\pi D} /$ $z F D \bar{c} \sqrt{a}$ vs. at in Fig. 5 shows the transient for progressive nucleation for different theoretical models.

The properties of the transient are summarised in Tables 1 and 2.

The current maximum is some $20 \%$ higher than predicted by the Scharifker-Mostany model but lower than predicted by Mirkin and Nilov [6] and Heerman and Tarallo [8] and the current maximum occurs at slightly shorter times. The present model still predicts an "overgrow" of the Cottrell line but of course much less pronounced than the Mirkin-Nilov [6] equation. Since $j_{\max } \sqrt{\pi D t_{\max }} / z F D \bar{c}$ is close to unity, the Cottrell line passes almost through the transient at the position of the 

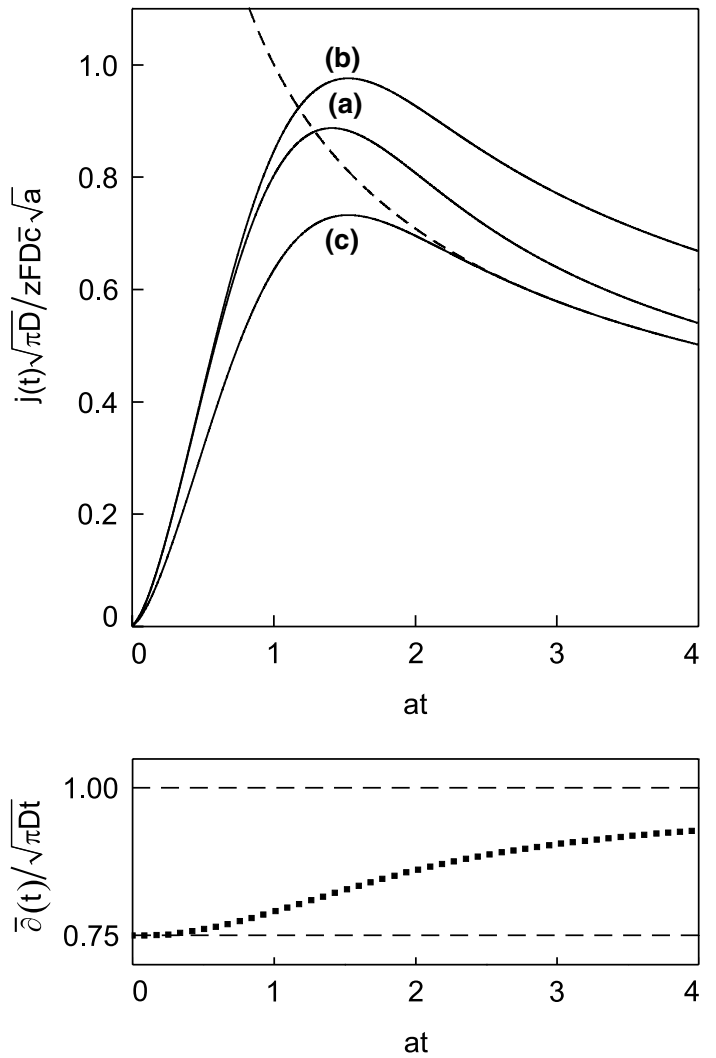

Fig. 5. (a) Dimensionless plot of the potentiostatic transient for progressive nucleation (this work) (b) Plot of $j(t) \sqrt{\pi D} / z F D \bar{c} \sqrt{a}=$ $(4 / 3) 1 / \sqrt{a t}\left[1-\exp \left(-a^{2} t^{2}\right)\right]$ according to the model of Mirkin-Nilov [6] and Heerman-Tarallo [8]. (c) Standard model of Scharifker-Mostany [3]. The lower figure shows a plot of $\bar{\partial}(t) / \sqrt{\pi D t}$ vs. at, i.e. the ratio of the thickness of the (hypothetical) uniform diffusion layer to the diffusion layer thickness for planar diffusion (Cottrell).

Table 1

Properties of the potentiostatic transient for progressive nucleation

\begin{tabular}{llll}
\hline & SM & This work & MNHT \\
\hline$a t_{\max }$ & 1.5286 & 1.4127 & 1.5286 \\
$j_{\max } \sqrt{\pi D} / z F D \bar{c}$ & 0.7306 & 0.8856 & 0.9741 \\
$j_{\max } \sqrt{\pi D t_{\max }} / z F D \bar{c}$ & 0.9034 & 1.0526 & 1.2042 \\
\hline
\end{tabular}

(1) $a=\sqrt{\pi D k A N_{0} / 2}=\sqrt{\pi} k D N_{\mathrm{S}}$ with $N_{\mathrm{S}}=\sqrt{A N_{0} / 2 k D}$. (2) $\mathrm{SM}$ refers to the model of Scharifker and Mostany [3]; MNHT refers to the model proposed by Mirkin and Nilov [6] and Heerman and Tarallo $[8,9]$.

current maximum. In principle the value of $a=\sqrt{\pi k D A N_{0} / 2}$ can be determined simply from the position of the maximum (see the first entry of Table 1). Then it follows that such an analysis according to the Scharifker-Mostany model or the Mirkin-Nilov model gives values of $I_{\mathrm{st}}=A N_{0}$ and $N_{\mathrm{S}}$ that are higher by $17 \%$ and $8 \%$, respectively than follows from an analysis according to the present model (with the evident condition that the same value of the, supposedly known, diffusion coefficient is used). However, such an analysis is based on the properties of a single experimental point and is
Table 2

Numerical data of $j(t) \sqrt{\pi D} / z F D \bar{c} \sqrt{a t}$ vs. at

\begin{tabular}{ll}
\hline$a t$ & $j(t) \sqrt{\pi D} / z F D \bar{c} \sqrt{a}$ \\
\hline 0 & 0 \\
0.1 & 0.0422 \\
0.2 & 0.1170 \\
0.3 & 0.2089 \\
0.4 & 0.3093 \\
0.5 & 0.4115 \\
0.6 & 0.5101 \\
0.7 & 0.6008 \\
0.8 & 0.6804 \\
0.9 & 0.7471 \\
1.0 & 0.7999 \\
1.1 & 0.8391 \\
1.2 & 0.8654 \\
1.3 & 0.8804 \\
1.4 & 0.8856 \\
1.5 & 0.8829 \\
1.6 & 0.8741 \\
1.7 & 0.8607 \\
1.8 & 0.8442 \\
1.9 & 0.8258 \\
2.0 & 0.8063 \\
2.2 & 0.7670 \\
2.4 & 0.7296 \\
2.6 & 0.6955 \\
2.8 & 0.6650 \\
3.0 & 0.6379 \\
3.2 & 0.6137 \\
3.4 & 0.5920 \\
3.6 & 0.5724 \\
3.8 & 0.5546 \\
4.0 & 0.5384 \\
4.2 & 0.5235 \\
4.6 & 0.5098 \\
4.8 & 0.4971 \\
5.0 & 0.4853 \\
\hline & 0.4743 \\
\hline
\end{tabular}

not recommended. The initial rising part of the transient can be described with very good precision by a 3-order polynomial, i.e.

$\frac{j(t) \sqrt{\pi D}}{z F D \bar{c}}=\sqrt{a}\left[a_{1}+a_{2}(a t)+a_{3}(a t)^{2}+a_{4}(a t)^{3}\right]$,

where $a_{1}=-0.00775, \quad a_{2}=0.39175, \quad a_{3}=1.39647$, $a_{4}=-1.01491$. Eq. (26) describes the transient for at $\leqslant 0.85$ (this maximum value corresponds to currents that are roughly $75 \%$ of the current at the maximum).

\section{Overlap function}

The current density corresponding with the growth of the real nuclei for the hypothetical situation of independent growth is given by

$j^{*}(t)=\int_{0}^{t} I(t, u) \frac{\mathrm{d} N_{\mathrm{a}}(u)}{\mathrm{d} u} \mathrm{~d} u$.

Similarly, the current density can be written as 
$j(t)=\int_{0}^{t} \tilde{I}(t, u) \frac{\mathrm{d} N_{\mathrm{a}}(u)}{\mathrm{d} u} \mathrm{~d} u$,

where $\tilde{I}(t, u)$ is the current to a single nucleus born at time $u$ that starts growing under the influence of the depletion zones associated with the already existing nuclei. With Eq. (25), the equation for the current density can be written as

$$
\begin{aligned}
j(t) & =j^{*}(t) \frac{\theta(t)}{\theta^{*}(t)}=j^{*}(t) F(t) \\
& =\int_{0}^{t} F(t) I(t, u) \frac{\mathrm{d} N_{\mathrm{a}}(u)}{\mathrm{d} u} \mathrm{~d} u,
\end{aligned}
$$

where $F(t)=\theta(t) / \theta^{*}(t)$ is the "overlap function" that operates on $j^{*}(t)$ to describe the effect of the interference of diffusion fields. Comparison of Eqs. (27) and (28) shows that the current to a single nucleus in the case of overlap is given by

$\tilde{I}(t, u)=F(t) I(t, u)=z F D \bar{c} \sqrt{\pi D} k \frac{\theta(t)}{\theta^{*}(t)} \sqrt{t-u}$.

Thus, the overlap model presented here implies that the overlap function $F(t)$, shown in Fig. 6, also describes the
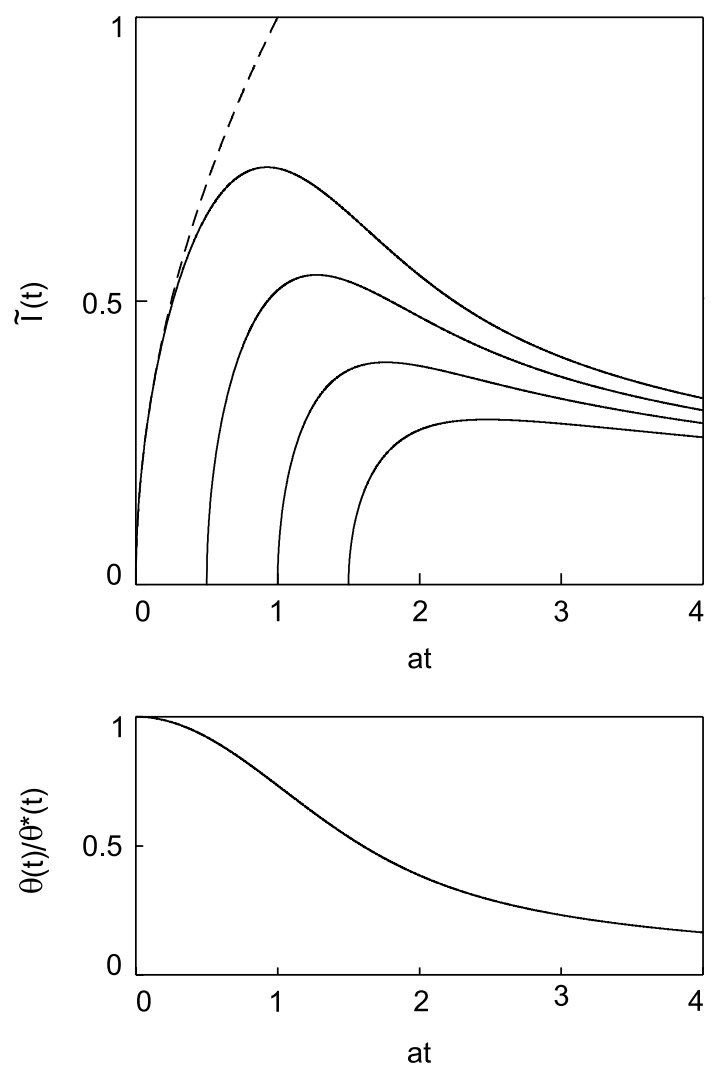

Fig. 6. Plot of the current (relative scale) to a single nucleus born at different times in the case of overlap of the diffusion fields (the dotted line shows the current to a single nucleus, born at $t=0$, that grows independently). The lower figure shows a plot of the "overlap function" $\theta(t) / \theta^{*}(t)$ vs. at. effect of overlap on the current to a single nucleus from the moment of its birth.

Integration of Eq. (29) gives the charge associated with a single growing nucleus which evidently is related to its size. In principle this can be coupled with the appearance rate of the real nuclei, Eq. (6), and this would allow a theoretical calculation of the size distribution of the clusters. However, in reality the size distribution depends not only on the appearance rate of the nuclei but also on the spatial distribution of the clusters over the electrode surface. This last effect is not accounted for by Eq. (29) and integration of this equation would yield the average size of the nuclei born at a given time. Therefore, it seems questionable whether the theoretical size distribution so obtained can be compared directly with experiment.

\section{Experimental}

A series of transients was recorded for the nucleation of silver from $0.1 \mathrm{M} \mathrm{AgNO}_{3}+2.5 \mathrm{M} \mathrm{KNO}_{3}+0.3 \mathrm{M}$ $\mathrm{HNO}_{3}$ solution at $37{ }^{\circ} \mathrm{C}$. The working electrode was a polished 2-mm diameter glassy carbon disk (geometrical area: $0.0314 \mathrm{~cm}^{2}$ ). The counter electrode was a pure silver foil and the reference electrode was a pure silver wire positioned via a Luggin-capillary close to the working electrode. All experiments were performed at the same overpotential, $\eta=-0.112 \mathrm{~V}$. After each experiment the silver clusters were dissolved in nitric acid to maintain a constant surface state of the electrode (it has been shown previously that anodic dissolution leads to an increased activity of the electrode [23]). The electrochemical cell included an optically flat window for the in situ microscopic observation and counting of the growing clusters. Experiments were performed using the double pulse technique with different durations of the nucleation pulse, allowing the recording of the experimental $N(t)$ vs. time curve by counting the number of clusters on the electrode surface after the end of the growth pulse [41].

\section{Experimental results}

A series of experimental transients is shown in Fig. 7. These transients in this figure were selected to give an idea of the reproducibility of the experiments. However, many transients situated somewhere in the middle between the extremes were omitted to avoid "overcrowding" of the figure that would appear otherwise just as a big black spot. It is evident that there are rather large variations for the current at the maximum but higher values of the current maximum correspond systematically with lower values of $t_{\max }$. Fig. 7 clearly indicates that it is dangerous, if not impossible, to draw definite 
conclusions from single transients as discussed previously [42]. At longer times, after the maximum, the current does not decay according to the Cottrell equation but almost tends to a constant value. These deviations from Cottrell behaviour at longer times can be attributed to edge effects caused by the finite, rather small size of the electrode.

Dimensionless plots of $\left(j(t) / j_{\max }\right)^{2}$ vs. $t / t_{\max }$ for different experimental transients are shown in Fig. 8 with the corresponding plots for the limiting cases of instantaneous and progressive nucleation. This figure indicates that the nucleation of silver on the glassy carbon disk at the overpotential of the experiments corresponds to progressive nucleation (the deviation of the theoretical line for $t / t_{\max } \geqslant 1.5$ corresponds to the deviation of Cottrell behaviour at longer times) (see Fig. 7).

The experimental $N(t)$ vs. time curve determined by optical counting of the clusters for times up to $4 \mathrm{~s}$ is shown in Fig. 9. Eq. (10) can be written in the form

$N(t)=\frac{1}{\sqrt{\pi} k D} a \operatorname{erf}(a t)$

so that fitting of the experimental $N(t)$ vs. time curve is possible with only one adjustable parameter, on condition that the value of the diffusion coefficient is known. The value $D=3.00 \times 10^{-5} \mathrm{~cm}^{2} \mathrm{~s}^{-1}$ was estimated from the current at longer times and is in good agreement with earlier reports [43,44]. Then, with $k=0.1607$,

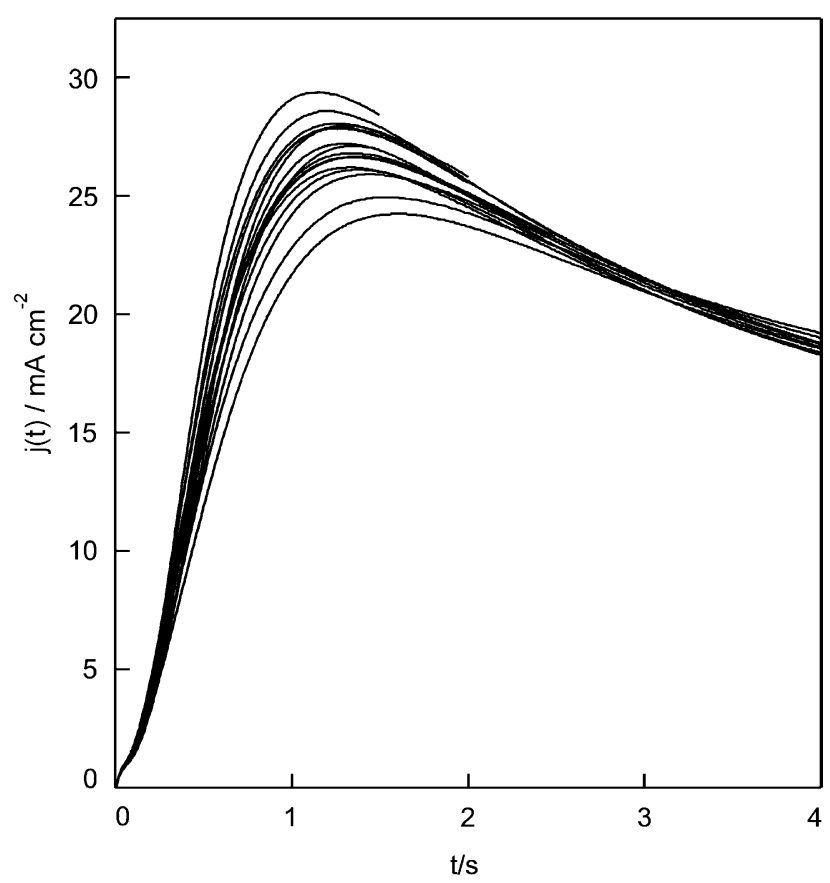

Fig. 7. Transients recorded for the nucleation of silver from $0.1 \mathrm{M}$ $\mathrm{AgNO}_{3}+2.5 \mathrm{M} \mathrm{KNO}_{3}+0.3 \mathrm{M} \mathrm{HNO}_{3}$ solution at $37{ }^{\circ} \mathrm{C}$ on a $2-\mathrm{mm}$ diameter glassy carbon disk (geometrical area: $0.0314 \mathrm{~cm}^{2}$. All transients were recorded at the same overpotential, $\eta=-0.112 \mathrm{~V}$ but for different times.

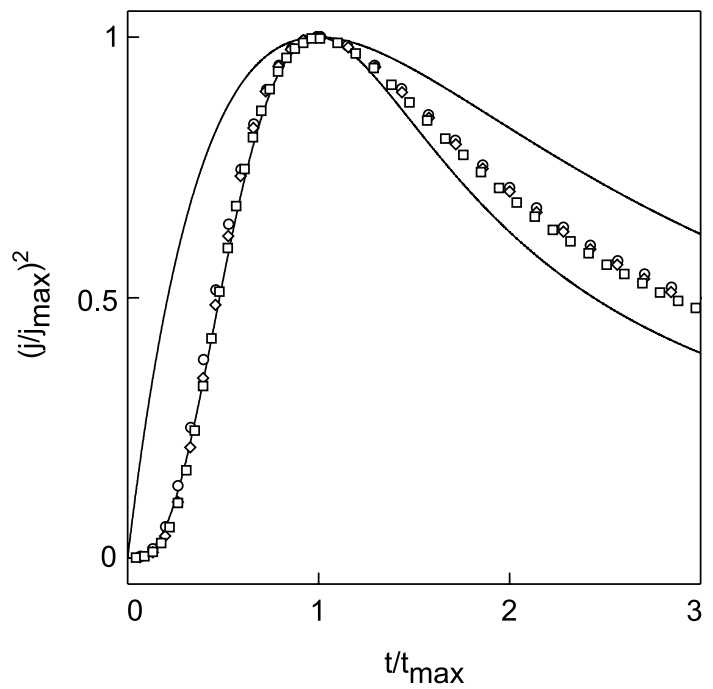

Fig. 8. Dimensionless plots of $\left(j(t) / j_{\max }\right)^{2}$ vs. $t / t_{\max }$ for different experimental transients. The solid lines correspond to the limiting cases of instantaneous and progressive nucleation.

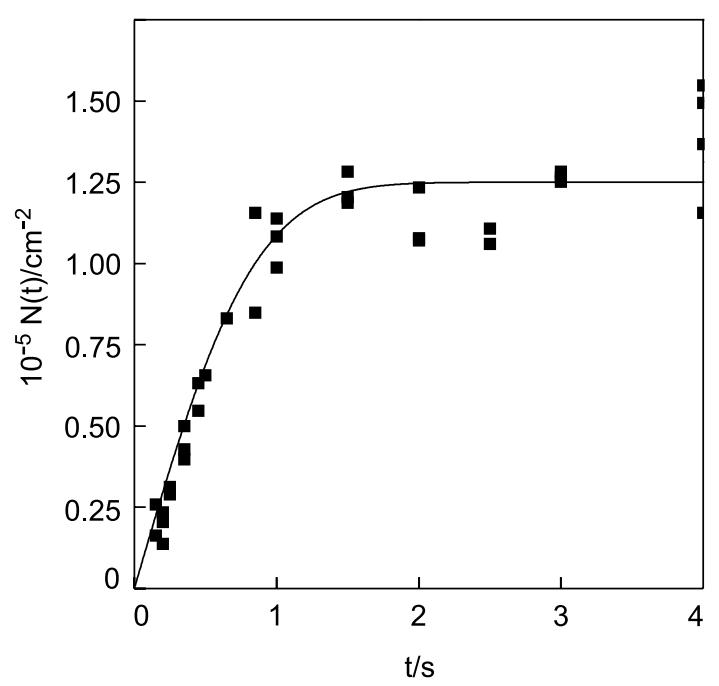

Fig. 9. Experimental $N(t)$ vs. time relation determined by optical counting of the clusters on the electrode (solid squares). The solid line was calculated using Eq. (10) with $N_{\mathrm{S}}=1.25 \times 10^{5} \mathrm{~cm}^{-2}$ $\left(I_{\mathrm{st}}=A N_{0}=1.51 \times 10^{5} \mathrm{~cm}^{-2} \mathrm{~s}^{-1}, \quad D=3.0 \times 10^{-5} \mathrm{~cm}^{-2} \mathrm{~s}^{-1}, \quad k=\right.$ $0.1607), a=1.0681 \mathrm{~s}^{-1}$

$a=1.10 \pm 0.02 \mathrm{~s}^{-1}, N_{\mathrm{S}}=1.25 \times 10^{5} \pm 3 \times 10^{3} \mathrm{~cm}^{-2}$ and $I_{\text {st }}=A N_{0}=1.55 \times 10^{5} \pm 5 \times 10^{3} \mathrm{~cm}^{-2} \mathrm{~s}^{-1}$.

The current transients were analysed by fitting the experimental data to the theoretical polynomial, Eq. (26). Some typical examples are shown in Figs. 10 and 11. As can be seen from these figures, the polynomial approximation fits the experimental data very well, over the range of its validity, with values of the nucleation rate constant and the nucleus saturation density very close to the values determined by direct optical counting.

The theoretical model describes reasonably well the position of the maximum but slightly overestimates the 


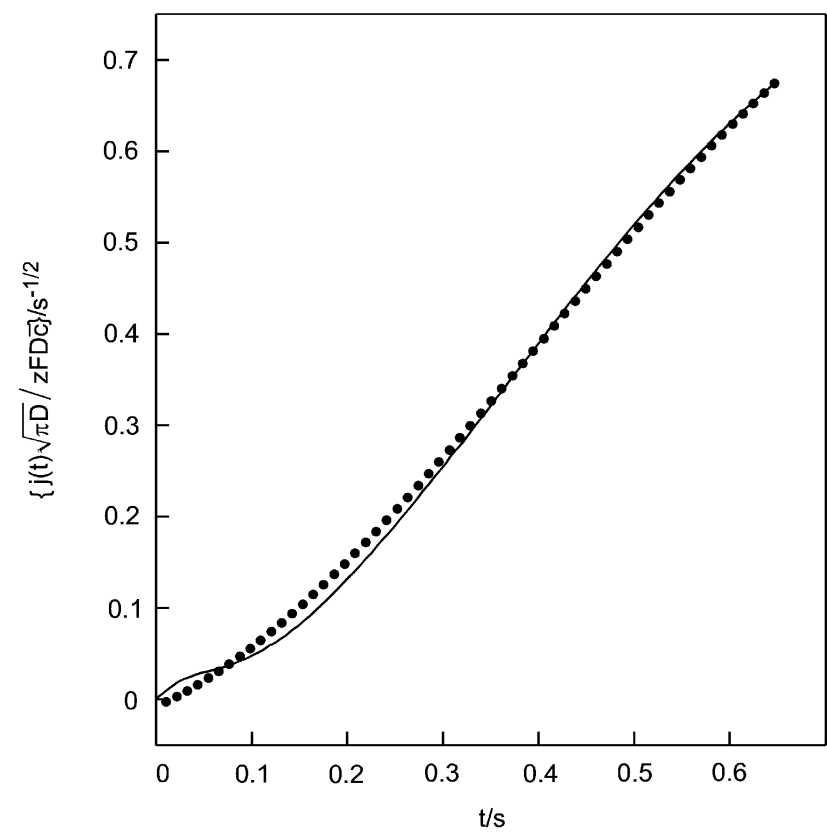

Fig. 10. Experimental transient (solid line) recorded during $0.65 \mathrm{~s}$ (optically determined number of nuclei: $N=8.31 \times 10^{4} \mathrm{~cm}^{-2}$ ). The polynomial fit (solid squares), Eq. (26), yields $a=1.1374 \pm 0.0019 \mathrm{~s}^{-1}$ $\left(I_{\mathrm{st}}=A N_{0}=1.71 \times 10^{5} \pm 6 \times 10^{2} \mathrm{~cm}^{-2} \mathrm{~s}^{-1}, N_{\mathrm{S}}=1.33 \times 10^{5} \pm 3 \times 10^{2}\right.$ $\left.\mathrm{cm}^{-2}, N=9.37 \times 10^{4} \mathrm{~cm}^{-2}\right)$.

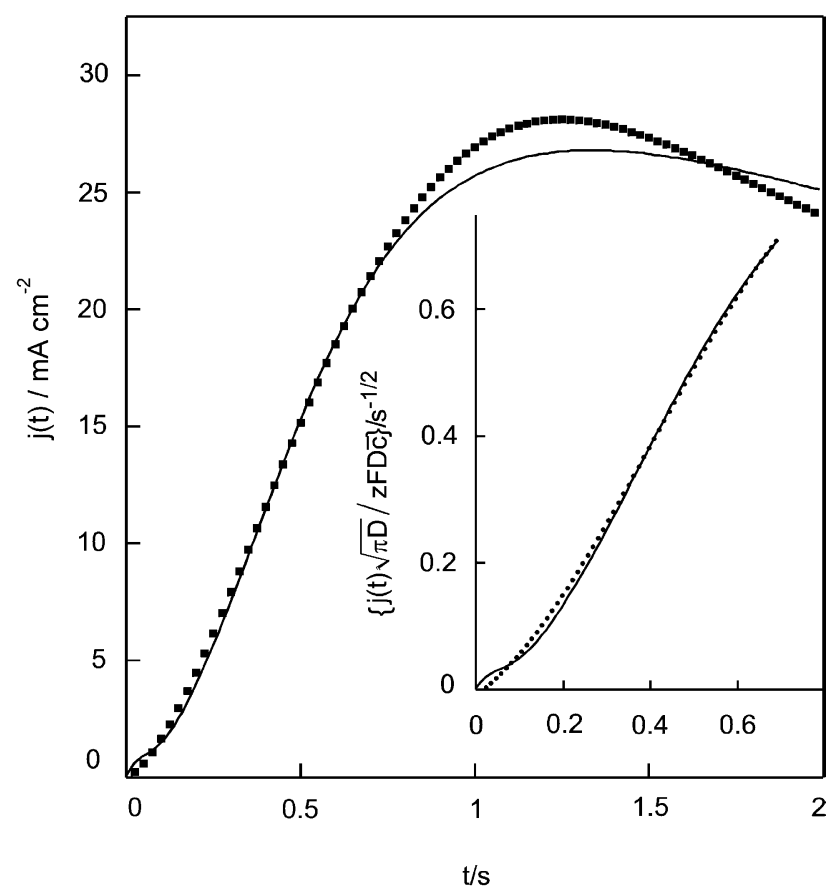

Fig. 11. Experimental transient (solid line) and theoretical fit (solid squares) calculated with $a=1.1303 \pm 0.0020 \mathrm{~s}^{-1} \quad\left(I_{\mathrm{st}}=A N_{0}=1.69 \times\right.$ $\left.10^{5} \pm 7 \times 10^{2} \mathrm{~cm}^{-2} \mathrm{~s}^{-1}, N_{\mathrm{S}}=1.32 \times 10^{5} \pm 3 \times 10^{2} \mathrm{~cm}^{-2}\right)$. This value of $a$ was determined from the polynomial fit, Eq. (26) (inset). The nucleus saturation density determined by optical counting is $N_{\mathrm{S}}=1.42 \times 10^{5} \mathrm{~cm}^{-2}$.

corresponding current. As pointed out already, analysis of experimental transients with the different theoretical models proposed thus far will yield very similar values of the stationary nucleation rate and the nucleus saturation density. However, it is clear that the region of the current maximum is the most of in all these models for the description of the current. The model proposed here allows a determination of the nucleation parameters while avoiding the use of the current maximum for the analysis of the transients.

\section{Conclusions}

This paper proposes a modification of the theoretical model of Mirkin and Nilov [6] and Heerman and Tarallo [8] for the potentiostatic transient for 3D nucleation with diffusion-controlled growth. This model leads to physically unacceptable results in the limit of long times for high values of the site density and low values of the nucleation rate constant (progressive nucleation). The model must be modified to take into account the formation of a finite number of nuclei due to the development of exclusion zones around the already existing nuclei. Then the equation for the transient predicts correctly the current in the limit of both very short and long times. In this paper only the case of progressive nucleation has been considered. The modification proposed here, Eq. (25), applies to the general case as well and this will be investigated in future work.

\section{Acknowledgements}

The authors (E.M., S.L., L.H.) thank the FWOVlaanderen for financial support. This study was carried out within the framework of the collaboration between the FWO-Vlaanderen and the Bulgarian Academy of Sciences. The experimental results were obtained with financial support of the Bulgarian Ministry of Education and Science (contract X-912-2000) using equipment donated to Prof. A. Milchev by the Alexander von Humboldt Foundation (Germany). One of the authors (S.L.) is indebted to the FWO-Vlaanderen for the award of a doctoral fellowship (Aspirant-FWO).

\section{References}

[1] G.A. Gunawardena, G.J. Hills, I. Montenegro, B.R. Scharifker, J. Electroanal. Chem. 138 (1982) 225.

[2] B.R. Scharifker, G. Hills, Electrochim. Acta 28 (1983) 879.

[3] B.R. Scharifker, J. Mostany, J. Electroanal. Chem. 177 (1984) 13.

[4] M. Sluyters-Rehbach, J.H.O.J. Wijenberg, E. Bosco, J. Sluyters, J. Electroanal. Chem. 236 (1987) 1.

[5] P.A. Bobbert, M.M. Wind, J. Vlieger, Physica 146A (1987) 69.

[6] M.V. Mirkin, A.P. Nilov, J. Electroanal. Chem. 283 (1990) 35.

[7] B.R. Scharifker, J. Mostany, M. Palomar-Pardavé, I. Gonzalez, J. Electrochem. Soc. 146 (1999) 1005.

[8] L. Heerman, A. Tarallo, J. Electroanal. Chem. 470 (1999) 70.

[9] L. Heerman, A. Tarallo, Electrochem. Commun. 2 (2000) 85. 
[10] P.C.T. D’Ajello, M.L. Munford, A.A. Pasa, J. Chem. Phys. 111 (1999) 4267.

[11] C. Johans, R. Lahtinen, K. Kontturi, D.J. Schiffrin, J. Electroanal. Chem. 488 (2000) 99.

[12] L. Heerman, E. Matthijs, S. Langerock, Electrochim. Acta 47 (2001) 905 .

[13] C. Amatore, in: I. Rubinstein (Ed.), Physical Electrochemistry. Principles, Methods and Applications, Marcel Dekker, New York, 1995, p. 131.

[14] A. Milchev, Electrocrystallization. Fundamentals of Nucleation and Growth, Kluwer Academic Publishers, Boston, 2002.

[15] W.S. Kruijt, Ph.D. Thesis, Utrecht, 1993.

[16] A. Milchev, W.S. Kruijt, M. Sluyters-Rehbach, J.H. Sluyters, J. Electroanal. Chem. 362 (1993) 21.

[17] W.S. Kruijt, M. Sluyters-Rehbach, J.H. Sluyters, A. Milchev, J. Electroanal. Chem. 371 (1994) 13.

[18] I. Markov, D. Kashchiev, J. Cryst. Growth 13-14 (1972) 131.

[19] I. Markov, D. Kashchiev, J. Cryst. Growth 16 (1972) 170.

[20] I. Markov, Thin Solid Films 35 (1976) 11.

[21] A. Milchev, Electrochim. Acta 28 (1983) 947.

[22] I. Markov, E. Stoycheva, Thin Solid Films 35 (1976) 21.

[23] A. Milchev, E. Vassileva, V. Kertov, J. Electroanal. Chem. 107 (1980) 323.

[24] A. Milchev, E. Vassileva, J. Electroanal. Chem. 107 (1980) 337.

[25] A. Serruya, J. Mostany, B.R. Scharifker, J. Chem. Soc., Faraday Trans. 89 (1993) 255.

[26] A. Serruya, J. Mostany, B.R. Scharifker, J. Electroanal. Chem. 464 (1999) 39.
[27] C. Ji, G. Oskam, P.C. Searson, J. Electrochem. Soc. 148 (2001) C746.

[28] A. Radisic, A.C. West, P.C. Searson, J. Electrochem. Soc. 149 (2001) C94.

[29] E. García-Pastoriza, J. Mostany, B.R. Scharifker, J. Electroanal. Chem. 441 (1998) 13.

[30] V. Tsakova, A. Milchev, J. Electroanal. Chem. 451 (1998) 211.

[31] A.N. Kolmogorov, Bull. Acad. Sci. URSS Sci. Math. Nat. 3 (1937) 355.

[32] M. Avrami, J. Chem. Phys. 7 (1939);

J. Chem. Phys. 8 (1940) 212;

J. Chem. Phys. 9 (1941) 177.

[33] U.R. Evans, Trans. Faraday Soc. 41 (1945) 365.

[34] V. Sessa, M. Fanfoni, M. Tomellini, Phys. Rev. B 54 (1996) 836.

[35] M. Tomellini, M. Fanfoni, Phys. Rev. B 55 (1997) 14071.

[36] M. Fanfoni, M. Tomellini, Nuovo Cimento 20D (1998) 1171.

[37] W.H. Mulder, J.H.O.J. Wijenberg, M. Sluyters-Rehbach, J.H. Sluyters, J. Electroanal. Chem. 270 (1989) 7.

[38] P. Uebele, H. Hermann, Modell. Simul. Mater. Sci., Eng. 4 (1996) 203.

[39] E. Pineda, D. Crespo, Phys. Rev. B 60 (1999) 3104.

[40] Y. Cao, P.C. Searson, A.C. West, J. Electrochem. Soc. 148 (2001) C376.

[41] A. Milchev, B. Scharifker, G. Hills, J. Electroanal. Chem. 132 (1982) 277.

[42] A. Milchev, L. Heerman, Electrochim. Acta 48 (2003) 2903.

[43] E. Michailova, A. Milchev, Electrochim. Acta 37 (1992) 2233.

[44] A. Milchev, E. Michailova, R. Lacman, B. Mueller-Zuelow, Electrochim. Acta 38 (1993) 535. 